\title{
AN EXTENDED COPRAS MODEL FOR MULTIPLE ATTRIBUTE GROUP DECISION MAKING BASED ON SINGLE-VALUED NEUTROSOPHIC 2-TUPLE LINGUISTIC ENVIRONMENT
}

\author{
Guiwu $\mathrm{WEI}^{1}$, Jiang WU${ }^{2}$, Yanfeng $\mathrm{GUO}^{3^{*}}$, Jie $\mathrm{WANG}^{4}$, Cun $\mathrm{WEI}^{5}$ \\ ${ }^{1}$ School of Business, Sichuan Normal University - Chenglong Campus, Chengdu, Sichuan, China \\ ${ }^{2,5}$ School of Statistic, Southwestern University of Finance and Economics, Chengdu, Sichuan, China \\ ${ }^{3}$ Southwestern University of Finance and Economics, Chengdu, Sichuan, China \\ ${ }^{4}$ School of Management and Economics, University of Electronic Science and Technology of China, \\ Chengdu, Sichuan, China
}

Received 30 June 2019; accepted 02 November 2020

\begin{abstract}
In this article, we develop the COPRAS model to solve the multiple attribute group decision making (MAGDM) under single-valued neutrosophic 2-tuple linguistic sets (SVN2TLSs). Firstly, we introduce the relevant knowledge about SVN2TLSs in a nutshell, such as the definition, the operation laws, a few of fused operators and so on. Then, combine the traditional COPRAS model with SVN2TLNs, and structure as well as elucidate the computing steps of the SVN2TLNCOPRAS pattern. Furthermore, in this article, we propose a method for determining attribute weights in different situations relying on the maximizing deviation method with SVN2TLNs. Last but not least, a numerical example about assessing the safety of construction project has been designed. And for further demonstrating the advantage of the new designed method, we also select a number of existed methods to have comparisons.
\end{abstract}

Keywords: multiple attribute group decision making (MAGDM), single-valued neutrosophic 2-tuple linguistic sets (SVN2TLSs), COPRAS model, construction project.

JEL Classification: C43, C61, D81.

\section{Introduction}

Social development has driven changes in the construction industry. In the increasingly fierce market competition, the construction progress is largely affect the construction cycle. Taking all kinds of factors in the process of construction has a crucial significance for managers of construction progress to effective control of the project from the beginning to the end. Thus, choosing the right construction scheme becomes a common multi-attribute decision making (MADM) issue. There is no doubt that the proposition of fuzzy set theory proposed by

\footnotetext{
*Corresponding author. E-mail: guoyanfeng@swufe.edu.cn
} 
Zadeh (1965) in 1965 is groundbreaking. Afterwards, more and more evaluation tools, such as intuitionistic fuzzy set (IFS) (Atanassov, 1986; Wu et al., 2019, 2020), Pythagorean fuzzy set (PFS) (Garg, 2016; He et al., 2019; Zhang \& Xu, 2014), Neutrosophic set (NS) (Wang et al., 2010; Wang et al., 2019a; Wei et al., 2019b), Spherical fuzzy set (SFS) (Gundogdu \& Kahraman, 2019; Wei et al., 2019a) and 2-tuple linguistic set (2TLS) (Herrera \& Martinez, 2000; Wang et al., 2019b) have been proposed and applied in MAGDM problems. The NS theory proposed by Smarandache (1999) considers three dimensions: truth-membership, indeterminacy-membership and false-membership. Ye (2014) studied the cross-entropy under single-valued neutrosophic sets (SVNSs) to solve MADM. Sahin and Liu (2016) proposed the maximizing deviation method under SVNSs. Chen and Ye (2017) combined the Dombi operation with SVNSs to present some Dombi weighted aggregating operators. Fan, Fan, and Ye (2018) presented the cosine measure and weighted cosine measure of SVNSs to handle actual problems. Wang, Zhang, Wang, and Li (2018c) defined some dual generalized SVN number Bonferroni mean (DGSVNNBM) operator. To get more scientific evaluation results, some scholars and researchers combine the SVNSs and 2TLS to define some new aggregation operator to solve MADM. Wang, Wei, and Wei (2018b) defined the 2-tuple linguistic neutrosophic set (2TLNSs) and presented some Bonferroni mean operators. Garg and Nancy (2018) explored the prioritized weighted operators under 2TLNSs. Wang, Wei, and $\mathrm{Lu}$ (2018a) built the TODIM model for MADM problems with 2TLNSs. Wu, Wu, Zhou, Chen, and Guan (2018) proposed the SVN2TLSs and developed some Hamacher aggregation operators. Ju, Ju, and Wang (2018) defined some MSM operators under SVN2TLS.

In order to make decisions based on the evaluation information more conveniently, Zavadskas, Kaklauskas, and Sarka (1994) built the COPRAS method for MADM. Zavadskas, Kaklauskas, and Kvederyte (2001) applied the COPRAS method to multiple criteria analysis. Podvezko (2011) made a comparison between SAW method and COPRAS method. Bekar, Cakmakci, and Kahraman (2016) adopted the fuzzy COPRAS method. Liou, Tamosaitiene, Zavadskas, and Tzeng (2016) defined the new hybrid COPRAS-G MADM. Mahdiraji, Arzaghi, Stauskis, and Zavadskas (2018) designed the hybrid fuzzy BWM-COPRAS method. Roy, Sharma, Kar, Zavadskas, and Saparauskas (2019) proposed the COPRAS method for MADM problems. At the same time, some other researches also investigated the COPRAS method (Mahdiraji et al., 2018; Matic et al., 2019; Roy et al., 2019).

From above review and analysis, we could find that the SVN2TLSs can consider both quantitative and qualitative evaluation information to describe the decision environment more objectively, at the same time, as a simple and effective decision-making tool, the COPRAS model can be more easily applied to deal with MAGDM problems. Thus, it's of great significance to study the single-valued neutrosophic 2-tuple linguistic number COPRAS (SVN2TLN-COPRAS) model. The innovation of such work is to set up an extended COPRAS model for MAGDM under the SVN2TLSs more effectively.

Our article includes the following contents. Firstly, elementary knowledge about SVN2TLSs is briefly introduced in Section 1. Secondly, the computing steps of SVN2TLNCOPRAS are presented in Section 23. To determine the attribute's weight, the maximizing deviation methods with incompletely weight and completely unknown weight are simply designed in Section 3. What's more, the new MAGDM method combining the COPRAS 
model and the maximizing deviation method is testified by taking advantage of a numerical example about assessing the safety of construction project in Section 4. And for further demonstrating the advantage of the new designed method, we also select a number of existed methods in this Section. Ultimately, we summarize this article in last section.

\section{Preliminaries}

\subsection{Single-valued neutrosophic 2-tuple linguistic sets}

On the basis of single-valued neutrosophic and 2-tuple linguistic knowledge, Wu et al. (2018) originally developed the single-valued neutrosophic 2-tuple linguistic sets (SVN2TLSs) which means we are capable to analyze uncertainty more conveniently. The basic definition of SVN2TLSs can be described as:

Definition 1 (Wu et al., 2018). Suppose there is a linguistic term collection $\left\{\zeta_{1}, \zeta_{2}, \ldots, \zeta_{k}\right\}$. Every element $\zeta_{i}(i=1,2, \ldots, k)$ in this collection represents a possible linguistic scale, and $\zeta=\left\{\zeta_{0}=\right.$ exceedingly terrible, $\zeta_{1}=$ very terrible, $\zeta_{2}=$ terrible, $\zeta_{3}=$ medium, $\zeta_{4}=$ well, $\zeta_{5}=$ very well, $\zeta_{6}=$ exceedingly well $\}$, then the SVN2TLSs could be defined:

$$
\zeta=\left\langle\left(x_{\phi}, \varphi\right),(\eta, \tau, \lambda)\right\rangle
$$

where $x_{\phi} \in \zeta,\left(x_{\phi}, \varphi\right)$ means the 2-tuple linguistic variables which meets the condition $0 \leq \Delta^{-1}\left(\begin{array}{l}\phi \\ x_{\phi}\end{array}, \varphi\right) \leq k .(\eta, \tau, \lambda) \in[0,1]$ means the truth-membership (TM), the indeterminacy membership (IM) and the falsity membership (FM) which satisfies the condition $0 \leq \eta+\tau+\lambda \leq 3$.

Definition 2(Wu et al., 2018). Let $\zeta_{1}=\left\langle\left(x_{\phi_{1}}, \varphi_{1}\right),\left(\eta_{1}, \tau_{1}, \lambda_{1}\right)\right\rangle$ and $\zeta_{2}=\left\langle\left(x_{\phi_{2}}, \varphi_{2}\right),\left(\eta_{2}, \tau_{2}, \lambda_{2}\right)\right\rangle$ be two SVN2TLNs, then the operation rules of them can be presented as:

(1) $\zeta_{1} \oplus \zeta_{2}=\left\{\Delta\left(\Delta^{-1}\left(x_{\phi_{1}}, \varphi_{1}\right)+\Delta^{-1}\left(x_{\phi_{2}}, \varphi_{2}\right)\right),\left(\eta_{1}+\eta_{2}-\eta_{1} \eta_{2}, \tau_{1} \tau_{2}, \lambda_{1} \lambda_{2}\right)\right\}$;

(2) $\zeta_{1} \otimes \zeta_{2}=\left\{\Delta\left(\Delta^{-1}\left(x_{\phi_{1}}, \varphi_{1}\right) \times \Delta^{-1}\left(x_{\phi_{2}}, \varphi_{2}\right)\right),\left(\eta_{1} \eta_{2}, \tau_{1}+\tau_{2}-\tau_{1} \tau_{2}, \lambda_{1}+\lambda_{2}-\lambda_{1} \lambda_{2}\right)\right\} ;$

(3) $\delta \zeta_{1}=\left\{\Delta\left(\delta \Delta^{-1}\left(x_{\phi_{1}}, \varphi_{1}\right)\right),\left(1-\left(1-\eta_{1}\right)^{\delta},\left(\tau_{1}\right)^{\delta},\left(\lambda_{1}\right)^{\delta}\right)\right\} \delta>0$;

(4) $\left(\zeta_{1}\right)^{\delta}=\left\{\Delta\left(\Delta^{-1}\left(x_{\phi_{1}}, \varphi_{1}\right)\right)^{\delta},\left(\left(\eta_{1}\right)^{\delta}, 1-\left(1-\tau_{1}\right)^{\delta}, 1-\left(1-\lambda_{1}\right)^{\delta}\right)\right\} \delta>0$.

Definition 3(Wu et al., 2018). Let $\zeta_{1}=\left\langle\left(x_{\phi_{1}}, \varphi_{1}\right),\left(\eta_{1}, \tau_{1}, \lambda_{1}\right)\right\rangle$ and $\zeta_{2}=\left\langle\left(x_{\phi_{2}}, \varphi_{2}\right),\left(\eta_{2}, \tau_{2}, \lambda_{2}\right)\right\rangle$ be two SVN2TLNs, then the score function $e\left(\zeta_{1}\right), e\left(\zeta_{2}\right)$ and accuracy function $h\left(\zeta_{1}\right), h\left(\zeta_{2}\right)$ can be depicted as:

$$
\begin{aligned}
& e\left(\zeta_{1}\right)=\Delta^{-1}\left(x_{\phi_{1}}, \varphi_{1}\right) \cdot \frac{\left(2+\eta_{1}-\tau_{1}-\lambda_{1}\right)}{3}, e\left(\zeta_{2}\right)=\Delta^{-1}\left(x_{\phi_{2}}, \varphi_{2}\right) \cdot \frac{\left(2+\eta_{2}-\tau_{2}-\lambda_{2}\right)}{3} \\
& h\left(\zeta_{1}\right)=\Delta^{-1}\left(x_{\phi_{1}}, \varphi_{1}\right) \cdot\left(\eta_{1}-\lambda_{1}\right), h\left(\zeta_{2}\right)=\Delta^{-1}\left(x_{\phi_{2}}, \varphi_{2}\right) \cdot\left(\eta_{2}-\lambda_{2}\right) .
\end{aligned}
$$

Then according to score and accuracy function of $\zeta_{1}$ and $\zeta_{2}$, if $e\left(\zeta_{1}\right) \geq e\left(\zeta_{2}\right), \zeta_{1} \geq \zeta_{2}$; if $e\left(\zeta_{1}\right)=e\left(\zeta_{2}\right)$ then: (1) if $h\left(\zeta_{1}\right) \geq h\left(\zeta_{2}\right), \zeta_{1} \geq \zeta_{2}$; (2) if $h\left(\zeta_{1}\right)=h\left(\zeta_{2}\right), \zeta_{1}=\zeta_{2}$. 


\subsection{The normalized Hamming distance}

Definition 4. Both $\zeta_{1}=\left\langle\left(x_{\phi_{1}}, \varphi_{1}\right),\left(\eta_{1}, \tau_{1}, \lambda_{1}\right)\right\rangle$ and $\zeta_{2}=\left\langle\left(x_{\phi_{2}}, \varphi_{2}\right),\left(\eta_{2}, \tau_{2}, \lambda_{2}\right)\right\rangle$ are SVN2TLNs, then the single-valued neutrosophic 2-tuple linguistic normalized Hamming distance (SVN2TLNHD) could be presented:

$d_{\text {SVN2TLNHD }}\left(\zeta_{1}, \zeta_{2}\right)=\frac{1}{3 k}\left[\left(2+\eta_{1}-\tau_{1}-\lambda_{1}\right) \cdot \Delta^{-1}\left(x_{\phi_{1}}, \varphi_{1}\right)-\left(2+\eta_{2}-\tau_{2}-\lambda_{2}\right) \cdot \Delta^{-1}\left(x_{\phi_{2}}, \varphi_{2}\right)\right]$.

\subsection{The aggregation operators}

Definition 5 (Wu et al., 2018). Let $\zeta_{q}=\left\langle\left(x_{\phi_{q}}, \varphi_{q}\right),\left(\eta_{q}, \tau_{q}, \lambda_{q}\right)\right\rangle(q=1,2, \ldots, p)$ be a set of SVN2TLNs, the single-valued neutrosophic 2-tuple linguistic weighted average (SVN2TLWA) operator and the single-valued neutrosophic 2-tuple linguistic weighted geometric (SVN2TLWG) operator are defined:

$$
\text { SVN2TLWA }=\underset{q=1}{p} \kappa_{q} \zeta_{q}=\kappa_{1} \zeta_{1} \oplus \kappa_{2} \zeta_{2} \oplus \ldots \oplus \kappa_{p} \zeta_{p}
$$

and

$$
\left.\operatorname{SVN} 2 \mathrm{TLWG}={\underset{q=1}{p}}_{\left(\zeta_{q}\right.}\right)^{\kappa_{q}}=\left(\zeta_{1}\right)^{\kappa_{1}} \otimes\left(\zeta_{2}\right)^{\kappa_{2}} \otimes \ldots \otimes\left(\zeta_{p}\right)^{\kappa_{p}}
$$

where

and

$$
\text { SVN2TLWA }=\left\{\begin{array}{l}
\Delta\left(\sum_{q=1}^{p}\left(\kappa_{q} \Delta^{-1}\left(x_{\phi_{q}}, \varphi_{q}\right)\right)\right), \\
\left\{1-\prod_{q=1}^{p}\left(1-\eta_{q}\right)^{\kappa_{q}}, \prod_{q=1}^{p}\left(\tau_{q}\right)^{\kappa_{q}}, \prod_{q=1}^{p}\left(\lambda_{q}\right)^{\kappa_{q}}\right\}
\end{array}\right\rangle
$$

$$
\text { SVN2TLWG }=\left\{\begin{array}{l}
\Delta\left(\prod_{q=1}^{p}\left(\Delta^{-1}\left(x_{\phi_{q}}, \varphi_{q}\right)\right)^{\kappa_{q}}\right), \\
\left\{\prod_{q=1}^{p}\left(\eta_{q}\right)^{\kappa_{q}}, 1-\prod_{q=1}^{p}\left(1-\tau_{q}\right)^{\kappa_{q}}, 1-\prod_{q=1}^{p}\left(1-\lambda_{q}\right)^{\kappa_{q}}\right\}
\end{array}\right\rangle .
$$

\section{The SVN2TLN-COPRAS model for MAGDM}

In such section, the SVN2TLN-COPRAS model is designed for MAGDM. We define that the set of alternatives is $\left\{H_{1}, H_{2}, \ldots H_{g}\right\}$, the set of attributes is $\left\{T_{1}, T_{2}, \ldots T_{s}\right\}$ and the set of decision makers is $\left\{W_{1}, W_{2}, \ldots W_{p}\right\}$. Simultaneously, we assume that the weighting vector of these $s$ attributes is $\omega=\left(\omega_{1}, \omega_{2}, \ldots, \omega_{s}\right)^{T}, \omega_{z} \geq 0, \sum_{z=1}^{s} \omega_{z}=1$ and the weighting vector of these $p$ decision-makers is $\kappa=\left(\kappa_{1}, \kappa_{2}, \ldots, \kappa_{p}\right)^{T}, \kappa_{q} \geq 0, \sum_{q=1}^{p} \kappa_{l}=1$. 
Step 1. Based on the information of $p$ decision makers, establish the SVN2TLN evaluation matrix $R=\left[a_{f z}^{q}\right]_{g \times s}(f=1,2, \ldots, g, z=1,2, \ldots, s)$. Moreover, according to Eq. (7) and DM's weight, we can obtain the fused matrix $\tilde{R}=\left[a_{f z}\right]_{g \times s}(f=1,2, \ldots, g, z=1,2, \ldots, s)$. All of these matrices just like the following shows.

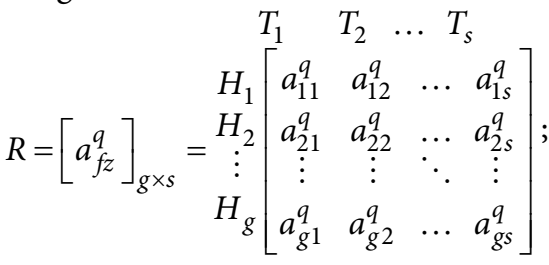

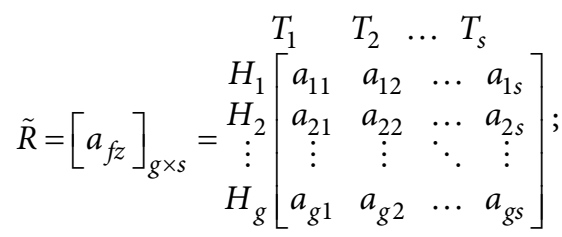

$$
\begin{aligned}
& a_{f z}=\kappa_{1} \phi_{f z}^{1} \oplus \kappa_{2} \phi_{f z}^{2} \oplus \cdots \oplus \kappa_{p} \phi_{f z}^{p} .
\end{aligned}
$$

Step 2. Based on the results of formula (11), we can obtain the normalized matrix $B=\left[b_{f z}\right]_{g \times s}$ by following formula:

$$
\begin{aligned}
& B=\left[b_{f z}\right]_{g \times s}=\begin{array}{c}
T_{1} \\
H_{1} \\
H_{2} \\
\vdots \\
H_{g}
\end{array}\left[\begin{array}{cccc}
b_{11} & b_{12} & \ldots & b_{1 s} \\
b_{21} & b_{22} & \ldots & b_{2 s} \\
\vdots & \vdots & \ddots & \vdots \\
b_{g 1} & b_{g 2} & \ldots & b_{g s}
\end{array}\right] ; \\
& b_{f z}=a_{f z} / \sum_{f=1}^{g} a_{f z}, f=1,2, \ldots, g, z=1,2, \ldots, s .
\end{aligned}
$$

Step 3. The Eq. (14) is useful in acquiring the corresponding weighted normalized matrix $C=\left[c_{f z}\right]_{g \times s}$ by making use of the information deriving from the normalized matrix $B=\left[b_{f z}\right]_{g \times s}$ and weight $\omega_{z}(z=1,2, \ldots, s)$.

$$
c_{f z}=\omega_{z} \otimes b_{f z}(f=1,2, \ldots, g, z=1,2, \ldots, s) .
$$

Step 4. According to each alternative's type, we can respectively calculate the sum value of benefit attributes $\Phi_{f(b)}$ and the sum value of cost attributes $\Phi_{f(c)}$, just as Eq. (15) and Eq. (16):

$$
\begin{gathered}
\Phi_{f(b)}=\sum_{z=1}^{k} c_{f z}, f=1,2, \ldots, g ; \\
\Phi_{f(c)}=\sum_{z=k+1}^{s} c_{f z}, f=1,2, \ldots, g,
\end{gathered}
$$

where $k$ is the number of the benefit attributes. Obviously, the best alternatives with bigger values of $\Phi_{f(b)}$ and smaller values of $\Phi_{f(c)}$. 
Step 5. Figure up the relative significance $Q_{f}$ of every alternative with the Eq. (17):

$$
Q_{f}=\Phi_{f(b)}+\frac{\left(\min \left(\Phi_{f(c)}\right) \times \sum_{f=1}^{g} \Phi_{f(c)}\right)}{\left(\Phi_{f(c)} \times \sum_{f=1}^{g} \frac{\min \left(\Phi_{f(c)}\right)}{\Phi_{f(c)}}\right)}=\Phi_{f(b)}+\frac{\sum_{f=1}^{g} \Phi_{f(c)}}{\left(\Phi_{f(c)} \times \sum_{f=1}^{g} \frac{1}{\Phi_{f(c)}}\right)}, f=1,2, \ldots, g
$$

where the optimal alternatives with bigger values of $Q_{f}$.

Step 6. Compute the values of utility degree $U_{f}$ based on each alternative's $Q_{f}$ by following equation:

$$
U_{f}=\frac{Q_{f}}{\max \left(Q_{f}\right)} \times 100 \%, f=1,2, \ldots, g .
$$

Step 7. The value of $U_{f}$ as a ranking criterion, the closer it is to $100 \%$, the better the corresponding alternative is. It's clear that the utility degree of best alternative is $100 \%$.

\section{Determine the attribute's weights}

It is almost impossible for decision makers to gather full information, which especially sticks out in the intricate decision making problems. To deal with such cases with incomplete attribute's weights, Wang (1998) proposed the maximizing deviation method which has been studied by a large amount of scholars (Wang et al., 2020; Yin et al., 2016) to handle MADM problems. Suppose the set of alternatives is $\left\{H_{1}, H_{2}, \ldots H_{g}\right\}$, the set of attributes is $\left\{T_{1}, T_{2}, \ldots T_{s}\right\}$ and the set of decision makers is $\left\{W_{1}, W_{2}, \ldots W_{p}\right\}$. Simultaneously, we assume that the weighting vector of these $s$ attributes is $\omega=\left(\omega_{1}, \omega_{2}, \ldots, \omega_{s}\right)^{T}, \omega_{z} \geq 0, \sum_{z=1}^{\infty} \omega_{z}=1$ and the weighting vector of these $p$ decision-makers is $\kappa=\left(\kappa_{1}, \kappa_{2}, \ldots, \kappa_{p}\right)^{T}, \kappa_{q} \geq 0, \sum_{q=1}^{p} \kappa_{l}=1$. Based on the above conditions, we can calculate difference degree between any two alternatives in line with the maximizing deviation method just as the Eq. (19) shows:

$$
D_{f z}^{(q)}(\omega)=\sum_{y=1}^{g} d\left(a_{f z}^{(q)}, a_{y z}^{(q)}\right) \omega_{z}, f=1,2, \ldots, g, z=1,2, \ldots, s,
$$

where $d\left(a_{f z}^{(q)}, a_{y z}^{(q)}\right)$ expresses the distance between $a_{f z}^{(q)}$ and $a_{y z}^{(q)}$, and $a_{f z}^{(q)}$ includes all evaluation information of $H_{f}$ with respect to $T_{z}$ deriving from the expert $W_{q}$.

Furthermore, the combined deviation of all alternatives $D_{z}^{(q)}(\omega)$ under attribute $T_{z}$ is calculated from different experts.

$$
D_{z}^{(q)}(\omega)=\sum_{f=1}^{g} D_{f z}^{(q)}(\omega)=\sum_{f=1}^{g} \sum_{y=1}^{g} d\left(a_{f z}^{(q)}, a_{y z}^{(q)}\right) \omega_{z}, z=1,2, \ldots, s .
$$

\subsection{The maximizing deviation method with SVN2TLNs}

In this section, the following nonlinear programming model is used to compute attribute's weight based on the maximizing deviation method. 
Case 1. The following non-linear programming model is for cases where the partial attribute weights are accessible.

$$
(M-1)\left\{\begin{array}{l}
\max D(\omega)=\sum_{z=1}^{s} \sum_{f=1}^{g} D_{f z}^{(q)}(\omega)=\sum_{m=1}^{q} \kappa_{q} \sum_{z=1}^{s} \sum_{f=1}^{g} \sum_{y=1}^{g} d\left(a_{f z}^{(q)}, a_{y z}^{(q)}\right) \omega_{z} \\
\text { subject to } \quad \omega_{z} \geq 0, \quad \sum_{z=1}^{s} \omega_{z}=1 \quad z=1,2, \ldots, s
\end{array},\right.
$$

where $\kappa_{q}$ depicts the weights of $W_{q}$ and

$$
d\left(a_{f z}^{(q)}, a_{y z}^{(q)}\right)=\frac{1}{3 k}\left[\begin{array}{l}
\left(2+\eta_{f z}^{(q)}-\tau_{f z}^{(q)}-\lambda_{f z}^{(q)}\right) \cdot \Delta^{-1}\left(x_{\phi}, \varphi\right)_{f z}^{(q)} \\
-\left(2+\eta_{y z}^{(q)}-\tau_{y z}^{(q)}-\lambda_{y z}^{(q)}\right) \cdot \Delta^{-1}\left(x_{\phi}, \varphi\right)_{y z}^{(q)}
\end{array} \mid\right]
$$

By solving the above equations, we can get the first-rank attribute weighting vector $\omega=\left(\omega_{1}, \omega_{2}, \ldots, \omega_{s}\right)^{T}$.

Case 2. For the cases where the entire attribute weights are unreachable, the following nonlinear programming model comes into play.

$$
(M-2)\left\{\begin{array}{c}
\max D(\omega)=\sum_{m=1}^{q} \kappa_{q} \frac{1}{3} \sum_{z=1}^{s} \sum_{f=1}^{g} \sum_{y=1}^{g}\left[\left|\frac{\left(2+\eta_{f z}^{(q)}-\tau_{f z}^{(q)}-\lambda_{f z}^{(q)}\right) \cdot \Delta^{-1}\left(x_{\phi}, \varphi\right)_{f z}^{(q)}}{k}\right|\right] \\
\text { subject to } \quad \omega_{z} \geq 0, \sum_{z=1}^{s} \omega_{z}^{2}=1 \quad z=1,2, \ldots, s
\end{array}\right] .
$$

The Lagrange function is necessary to build for solving this model, as follows:

$$
\begin{aligned}
& L(\omega, \pi)=\sum_{m=1}^{q} \kappa_{q} \frac{1}{3} \sum_{z=1}^{s} \sum_{f=1}^{g} \sum_{y=1}^{g}\left[\mid\left(\frac{\left(2+\eta_{f z}^{(q)}-\tau_{f z}^{(q)}-\lambda_{f z}^{(q)}\right) \cdot \Delta^{-1}\left(x_{\phi}, \varphi\right)_{f z}^{(q)}}{k}-\right.\right. \\
& \left.\frac{\left(2+\eta_{y z}^{(q)}-\tau_{y z}^{(q)}-\lambda_{y z}^{(q)}\right) \cdot \Delta^{-1}\left(x_{\phi}, \varphi\right)_{y z}^{(q)}}{k} \mid\right] \omega_{z}+\frac{\pi}{6}\left(\sum_{z=1}^{s} \omega_{z}^{2}-1\right),
\end{aligned}
$$

where $\pi$ is the Lagrange multiplier.

Next, take the partial derivatives of $\omega_{z}$ and $\pi$ in Eq. (24) respectively, and set the results equal to zero that we can get the following simultaneous equations. 


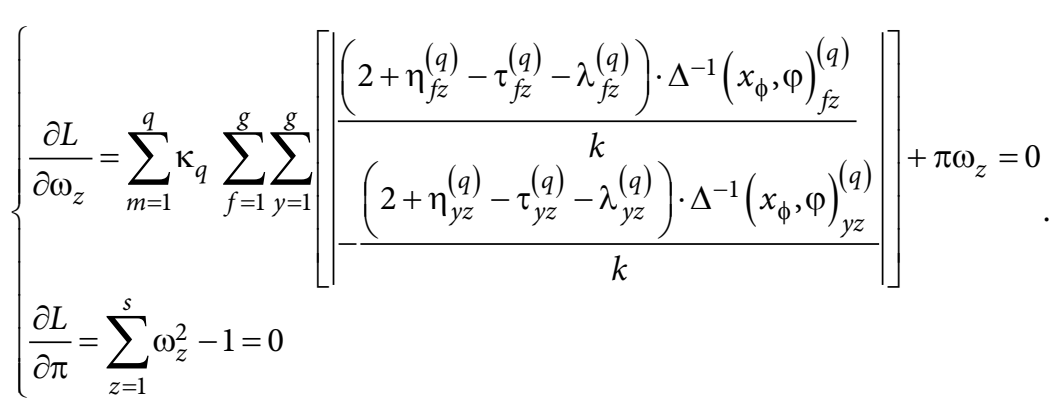

Finally, the weighting of attributes is worked out shown in Eq. (25) according to the partial derivatives and Eq. (24).

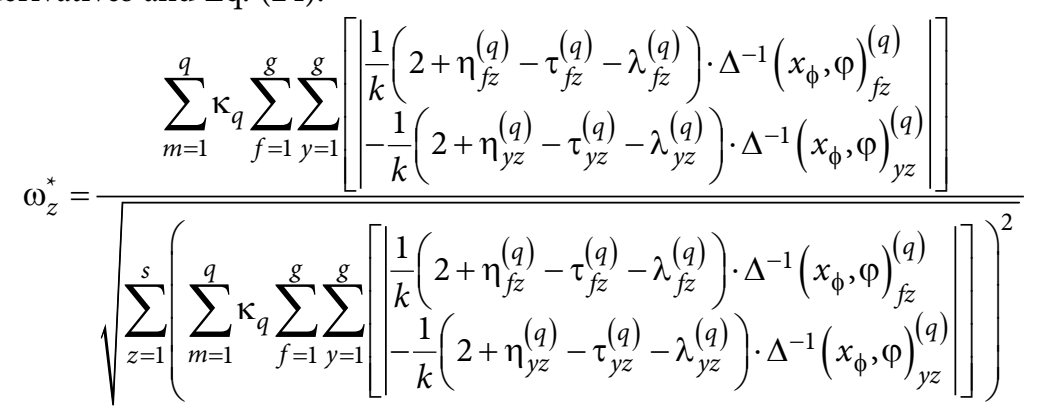

by normalizing the formula (25), the normalized results are listed as:

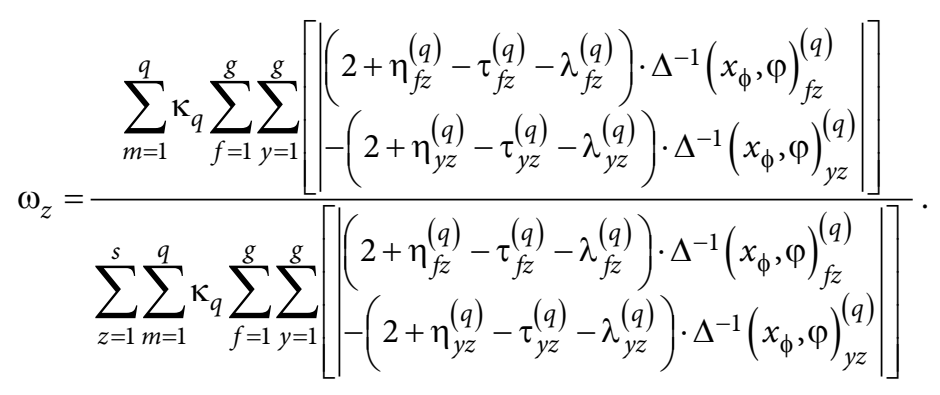

\subsection{The decision-making model}

To sum up, the SVN2TLN-COPRAS method for MAGDM issues for incompletely attribute's weights includes the following steps:

Step 1. Establish the SVN2TLN evaluation matrix $R=\left[a_{f z}^{q}\right]_{g \times s}$ where $a_{f z}^{q}$ demonstrates the SVN2TLN of alternatives $H_{f}(f=1,2, \ldots, g)$ in line with $T_{z}(z=1,2, \ldots, s)$ from expert $W_{q}$.

Step 2. Integrate all SVN2TLN decision matrices into the overall decision matrix $\tilde{R}=\left[a_{f z}\right]_{g \times s}$ with respect to Eq. (11).

Step 3. Take advantage of Eq. (21) or Eq. (26) respectively facing different cases to acquire the weighting of attributes. 
Step 4. Compute the corresponding weighted normalized matrix $C=\left[c_{f z}\right]_{g \times s}$ by using Eq. (14).

Step 5. According to $C=\left[c_{f z}\right]_{g \times s}$ and the types of attributes, the sum of benefit attributes $\Phi_{f(b)}$ and the sum of cost attributes $\Phi_{f(c)}$ are computed by Eq. (15) and Eq. (16).

Step 6. Figure up the relative significance $Q_{f}$ of every alternative with the Eq. (17).

Step 7. Compute the utility degree $U_{f}$ based on every alternative's $Q_{f}$ by Eq. (18).

Step 8. The value of $U_{f}$ as a ranking criterion, the closer it is to $100 \%$, the better the corresponding alternative is.

\section{Numerical example and comparative analysis}

\subsection{Numerical example}

The ultimate goal of the construction project is to complete the construction task with high quality and low consumption within the time limit specified in the contract, and to put it into production or deliver it for use on schedule. Any construction project, objectively there are a variety of technically feasible construction plans. The construction plan is to determine the construction sequence, construction method and selection of construction machinery according to the established construction deployment, so that the project can achieve the best effect of short duration, good quality and low cost. Therefore, there must be the problem of optimal selection of construction scheme. In most construction schemes, the quality of construction depends mainly on the following factors: (1) $T_{1}$ is the factor of management level of construction project construction unit; (2) $T_{2}$ is the construction of machinery, materials and other resource factors; (3) $T_{3}$ is a natural and social environmental factor; (4) $T_{4}$ is the influencing factor of relevant construction units; (5) $T_{5}$ is a variety of accident risk factors in the construction process; (6) $T_{6}$ is the technical level factor of the construction unit.

In order to consider both quantitative and qualitative evaluation information, in this section, a numerical example is provided to select best construction projects by COPRAS model with SVN2TLNs. The five possible construction projects $H_{f}(f=1,2,3,4,5)$ are to be assessed with SVN2TLNs under six criteria. In order to give reasonable evaluation information, we invite two practitioners $\left(W_{1}, W_{2}\right)$ with senior construction experience and one university professor $W_{3}$ with certain academic attainments in the field of construction to form an expert group to assess these construction projects. Moreover, according to the expert's age and authority in their field, through internal discussion, the expert's weight is $(0.35,0.25,0.40)$.

Step 1. Based on the information of $p$ decision makers, establish the SVN2TLN evaluation matrix $R=\left[a_{f z}^{q}\right]_{5 \times 6}($ See Tables $1-3)$. 
Table 1 . Evaluation matrix by $W_{1}$

\begin{tabular}{|c|c|c|c|}
\hline & $T_{1}$ & $T_{2}$ & $T_{3}$ \\
\hline$H_{1}$ & $\left\{\left(x_{2}, 0\right),(0.7,0.4,0.5)\right\}$ & $\left\{\left(x_{1}, 0\right),(0.6,0.4,0.3)\right\}$ & $\left\{\left(x_{3}, 0\right),(0.2,0.4,0.5)\right\}$ \\
\hline$H_{2}$ & $\left\{\left(x_{1}, 0\right),(0.6,0.5,0.1)\right\}$ & $\left\{\left(x_{4}, 0\right),(0.4,0.5,0.2)\right\}$ & $\left\{\left(x_{1}, 0\right),(0.5,0.1,0.4)\right\}$ \\
\hline$H_{3}$ & $\left\{\left(x_{3}, 0\right),(0.3,0.4,0.5)\right\}$ & $\left\{\left(x_{2}, 0\right),(0.5,0.4,0.6)\right\}$ & $\left\{\left(x_{4}, 0\right),(0.6,0.4,0.7)\right\}$ \\
\hline$H_{4}$ & $\left\{\left(x_{4}, 0\right),(0.8,0.1,0.2)\right\}$ & $\left\{\left(x_{5}, 0\right),(0.7,0.4,0.1)\right\}$ & $\left\{\left(x_{4}, 0\right),(0.6,0.2,0.3)\right\}$ \\
\hline$H_{5}$ & $\left\{\left(x_{5}, 0\right),(0.6,0.4,0.7)\right\}$ & $\left\{\left(x_{1}, 0\right),(0.5,0.1,0.3)\right\}$ & $\left\{\left(x_{4}, 0\right),(0.4,0.7,0.2)\right\}$ \\
\hline & $T_{4}$ & $T_{5}$ & $T_{6}$ \\
\hline$H_{1}$ & $\left\{\left(x_{1}, 0\right),(0.8,0.6,0.9)\right\}$ & $\left\{\left(x_{3}, 0\right),(0.5,0.1,0.4)\right\}$ & $\left\{\left(x_{4}, 0\right),(0.6,0.2,0.4)\right\}$ \\
\hline$H_{2}$ & $\left\{\left(x_{4}, 0\right),(0.5,0.1,0.4)\right\}$ & $\left\{\left(x_{1}, 0\right),(0.3,0.1,0.5)\right\}$ & $\left\{\left(x_{2}, 0\right),(0.5,0.3,0.6)\right\}$ \\
\hline$H_{3}$ & $\left\{\left(x_{5}, 0\right),(0.7,0.3,0.2)\right\}$ & $\left\{\left(x_{4}, 0\right),(0.6,0.1,0.4)\right\}$ & $\left\{\left(x_{3}, 0\right),(0.7,0.8,0.5)\right\}$ \\
\hline$H_{4}$ & $\left\{\left(x_{3}, 0\right),(0.5,0.3,0.1)\right\}$ & $\left\{\left(x_{5}, 0\right),(0.7,0.5,0.4)\right\}$ & $\left\{\left(x_{4}, 0\right),(0.4,0.1,0.2)\right\}$ \\
\hline$H_{5}$ & $\left\{\left(x_{4}, 0\right),(0.6,0.7,0.4)\right\}$ & $\left\{\left(x_{1}, 0\right),(0.4,0.6,0.5)\right\}$ & $\left\{\left(x_{1}, 0\right),(0.5,0.1,0.4)\right\}$ \\
\hline
\end{tabular}

Table 2. Evaluation matrix by $W_{2}$

\begin{tabular}{|c|c|c|c|}
\hline & $T_{1}$ & $T_{2}$ & $T_{3}$ \\
\hline$H_{1}$ & $\left\{\left(x_{3}, 0\right),(0.3,0.4,0.1)\right\}$ & $\left\{\left(x_{4}, 0\right),(0.5,0.7,0.2)\right\}$ & $\left\{\left(x_{1}, 0\right),(0.5,0.1,0.4)\right\}$ \\
\hline$H_{2}$ & $\left\{\left(x_{3}, 0\right),(0.2,0.4,0.5)\right\}$ & $\left\{\left(x_{1}, 0\right),(0.6,0.1,0.3)\right\}$ & $\left\{\left(x_{4}, 0\right),(0.3,0.1,0.2)\right\}$ \\
\hline$H_{3}$ & $\left\{\left(x_{1}, 0\right),(0.1,0.4,0.6)\right\}$ & $\left\{\left(x_{4}, 0\right),(0.6,0.4,0.1)\right\}$ & $\left\{\left(x_{3}, 0\right),(0.6,0.5,0.5)\right\}$ \\
\hline$H_{4}$ & $\left\{\left(x_{4}, 0\right),(0.3,0.1,0.2)\right\}$ & $\left\{\left(x_{3}, 0\right),(0.8,0.1,0.4)\right\}$ & $\left\{\left(x_{4}, 0\right),(0.7,0.1,0.3)\right\}$ \\
\hline$H_{5}$ & $\left\{\left(x_{3}, 0\right),(0.4,0.5,0.3)\right\}$ & $\left\{\left(x_{3}, 0\right),(0.3,0.1,0.2)\right\}$ & $\left\{\left(x_{3}, 0\right),(0.4,0.5,0.6)\right\}$ \\
\hline & $T_{4}$ & $T_{5}$ & $T_{6}$ \\
\hline$H_{1}$ & $\left\{\left(x_{4}, 0\right),(0.6,0.5,0.9)\right\}$ & $\left\{\left(x_{2}, 0\right),(0.5,0.1,0.7)\right\}$ & $\left\{\left(x_{4}, 0\right),(0.5,0.7,0.3)\right\}$ \\
\hline$H_{2}$ & $\left\{\left(x_{3}, 0\right),(0.4,0.3,0.2)\right\}$ & $\left\{\left(x_{3}, 0\right),(0.1,0.4,0.2)\right\}$ & $\left\{\left(x_{1}, 0\right),(0.6,0.4,0.1)\right\}$ \\
\hline$H_{3}$ & $\left\{\left(x_{1}, 0\right),(0.6,0.1,0.3)\right\}$ & $\left\{\left(x_{1}, 0\right),(0.4,0.5,0.3)\right\}$ & $\left\{\left(x_{3}, 0\right),(0.3,0.1,0.2)\right\}$ \\
\hline$H_{4}$ & $\left\{\left(x_{4}, 0\right),(0.5,0.1,0.2)\right\}$ & $\left\{\left(x_{4}, 0\right),(0.6,0.1,0.3)\right\}$ & $\left\{\left(x_{5}, 0\right),(0.6,0.2,0.3)\right\}$ \\
\hline$H_{5}$ & $\left\{\left(x_{5}, 0\right),(0.8,0.7,0.6)\right\}$ & $\left\{\left(x_{5}, 0\right),(0.2,0.3,0.4)\right\}$ & $\left\{\left(x_{1}, 0\right),(0.8,0.4,0.1)\right\}$ \\
\hline
\end{tabular}

Table 3. Evaluation matrix by $W_{3}$

\begin{tabular}{|c|c|c|c|}
\hline & $T_{1}$ & $T_{2}$ & $T_{3}$ \\
\hline$H_{1}$ & $\left\{\left(x_{3}, 0\right),(0.3,0.4,0.5)\right\}$ & $\left\{\left(x_{4}, 0\right),(0.6,0.4,0.3)\right\}$ & $\left\{\left(x_{1}, 0\right),(0.8,0.7,0.6)\right\}$ \\
\hline$H_{2}$ & $\left\{\left(x_{1}, 0\right),(0.1,0.5,0.6)\right\}$ & $\left\{\left(x_{1}, 0\right),(0.5,0.3,0.4)\right\}$ & $\left\{\left(x_{4}, 0\right),(0.5,0.6,0.2)\right\}$ \\
\hline$H_{3}$ & $\left\{\left(x_{4}, 0\right),(0.4,0.3,0.4)\right\}$ & $\left\{\left(x_{3}, 0\right),(0.6,0.2,0.1)\right\}$ & $\left\{\left(x_{2}, 0\right),(0.3,0.5,0.1)\right\}$ \\
\hline$H_{4}$ & $\left\{\left(x_{4}, 0\right),(0.8,0.3,0.4)\right\}$ & $\left\{\left(x_{3}, 0\right),(0.5,0.1,0.2)\right\}$ & $\left\{\left(x_{4}, 0\right),(0.6,0.1,0.4)\right\}$ \\
\hline$H_{5}$ & $\left\{\left(x_{3}, 0\right),(0.1,0.5,0.3)\right\}$ & $\left\{\left(x_{4}, 0\right),(0.3,0.5,0.3)\right\}$ & $\left\{\left(x_{1}, 0\right),(0.2,0.4,0.5)\right\}$ \\
\hline & $T_{4}$ & $T_{5}$ & $T_{6}$ \\
\hline$H_{1}$ & $\left\{\left(x_{3}, 0\right),(0.5,0.6,0.7)\right\}$ & $\left\{\left(x_{4}, 0\right),(0.3,0.4,0.7)\right\}$ & $\left\{\left(x_{3}, 0\right),(0.3,0.1,0.4)\right\}$ \\
\hline$H_{2}$ & $\left\{\left(x_{1}, 0\right),(0.3,0.2,0.4)\right\}$ & $\left\{\left(x_{3}, 0\right),(0.3,0.5,0.2)\right\}$ & $\left\{\left(x_{1}, 0\right),(0.6,0.3,0.7)\right\}$ \\
\hline$H_{3}$ & $\left\{\left(x_{4}, 0\right),(0.2,0.5,0.1)\right\}$ & $\left\{\left(x_{1}, 0\right),(0.1,0.4,0.2)\right\}$ & $\left\{\left(x_{4}, 0\right),(0.3,0.1,0.5)\right\}$ \\
\hline$H_{4}$ & $\left\{\left(x_{5}, 0\right),(0.3,0.4,0.2)\right\}$ & $\left\{\left(x_{4}, 0\right),(0.8,0.1,0.3)\right\}$ & $\left\{\left(x_{5}, 0\right),(0.6,0.3,0.5)\right\}$ \\
\hline$H_{5}$ & $\left\{\left(x_{3}, 0\right),(0.5,0.1,0.6)\right\}$ & $\left\{\left(x_{4}, 0\right),(0.2,0.5,0.7)\right\}$ & $\left\{\left(x_{3}, 0\right),(0.1,0.4,0.2)\right\}$ \\
\hline
\end{tabular}


Step 2. Take advantage of Eq. (21) or Eq. (26) respectively facing different cases to acquire the weighting of attributes.

Case 1. When the partial attribute weights are accessible:

$$
\left\{\begin{array}{l}
0.14 \leq \omega_{1} \leq 0.16,0.15 \leq \omega_{2} \leq 0.20,0.10 \leq \omega_{3} \leq 0.30 \\
0.10 \leq \omega_{4} \leq 0.15,0.10 \leq \omega_{5} \leq 0.35,0.12 \leq \omega_{6} \leq 0.18 \\
\omega_{z} \geq 0, z=1,2, \ldots, 6, \sum_{z=1}^{6} \omega_{z}=1
\end{array}\right\}
$$

The following non-linear programming model could be obtained according to $(M-1)$ :

$$
\left\{\begin{array}{l}
\max D(\omega)=3.8689 \omega_{1}+3.4178 \omega_{2}+4.0000 \omega_{3}+3.8833 \omega_{4}+4.1778 \omega_{5}+4.0922 \omega_{6} \\
\text { subject to } \omega_{z} \geq 0, \sum_{z=1}^{6} \omega_{z}=1, z=1,2, \ldots, 6
\end{array} .\right.
$$

Deal with such model; the weight of attributes is obtained as:

$\omega=(0.1400,0.1500,0.1000,0.1000,0.3500,0.1600)$.

Case 2. When the entire attribute weights are unreachable, the weighting of attributes could be obtained through Eq. (11):

$$
\omega=(0.1651,0.1458,0.1706,0.1657,0.1782,0.1746) .
$$

Step 3. According to Eq. (7) and expert's weight, we can fuse overall $a_{f z}^{q}(f=1,2,3,4,5$; $z=1,2,3,4,5,6)$ into $a_{f z}$, the fused results are shown in Table 4 .

Table 4. The fused results by SVN2TLWA operator

\begin{tabular}{|l|c|c|c|}
\hline & $T_{1}$ & $T_{2}$ & $T_{3}$ \\
\hline$H_{1}$ & $\left\{\left(x_{3},-0.25\right),(0.4336,0.4000,0.2847)\right\}$ & $\left\{\left(x_{3}, 0.25\right),(0.5675,0.4865,0.2603)\right\}$ & $\left\{\left(x_{2},-0.50\right),(0.6102,0.3080,0.4974)\right\}$ \\
\hline$H_{2}$ & $\left\{\left(x_{2},-0.30\right),(0.2948,0.4624,0.3597)\right\}$ & $\left\{\left(x_{2},-0.25\right),(0.5160,0.2321,0.3041)\right\}$ & $\left\{\left(x_{3}, 0.25\right),(0.4375,0.2048,0.2378)\right\}$ \\
\hline$H_{3}$ & $\left\{\left(x_{3},-0.30\right),(0.2813,0.3565,0.4874)\right\}$ & $\left\{\left(x_{3}, 0.10\right),(0.5771,0.3031,0.1565)\right\}$ & $\left\{\left(x_{3},-0.15\right),(0.4996,0.4729,0.2857)\right\}$ \\
\hline$H_{4}$ & $\left\{\left(x_{4}, 0.00\right),(0.6899,0.1552,0.2639)\right\}$ & $\left\{\left(x_{4},-0.50\right),(0.6807,0.1414,0.2144)\right\}$ & $\left\{\left(x_{4}, 0.00\right),(0.6383,0.1189,0.3366)\right\}$ \\
\hline$H_{5}$ & $\left\{\left(x_{4},-0.50\right),(0.3624,0.4729,0.3708)\right\}$ & $\left\{\left(x_{3},-0.10\right),(0.3565,0.1904,0.2603)\right\}$ & $\left\{\left(x_{2}, 0.45\right),(0.3268,0.4974,0.4238)\right\}$ \\
\hline & $T_{4}$ & $T_{5}$ & $T_{6}$ \\
\hline$H_{1}$ & $\left\{\left(x_{3},-0.15\right),(0.6322,0.5629,0.8139)\right\}$ & $\left\{\left(x_{3}, 0.05\right),(0.4280,0.1741,0.6086)\right\}$ & $\left\{\left(x_{4},-0.40\right),(0.4590,0.2350,0.3617)\right\}$ \\
\hline$H_{2}$ & $\left\{\left(x_{2}, 0.45\right),(0.3903,0.1938,0.3138)\right\}$ & $\left\{\left(x_{3},-0.50\right),(0.2356,0.3092,0.2515)\right\}$ & $\left\{\left(x_{1}, 0.25\right),(0.5771,0.3318,0.3409)\right\}$ \\
\hline$H_{3}$ & $\left\{\left(x_{3}, 0.20\right),(0.5088,0.2505,0.1747)\right\}$ & $\left\{\left(x_{2},-0.25\right),(0.3624,0.3058,0.2741)\right\}$ & $\left\{\left(x_{3}, 0.40\right),(0.4336,0.1682,0.3628)\right\}$ \\
\hline$H_{4}$ & $\left\{\left(x_{4}, 0.15\right),(0.4280,0.2291,0.1682)\right\}$ & $\left\{\left(x_{4}, 0.25\right),(0.7179,0.1495,0.3224)\right\}$ & $\left\{\left(x_{5},-0.25\right),(0.5573,0.1978,0.3325)\right\}$ \\
\hline$H_{5}$ & $\left\{\left(x_{4},-0.05\right),(0.6569,0.3214,0.5422)\right\}$ & $\left\{\left(x_{4},-0.40\right),(0.2555,0.4376,0.5291)\right\}$ & $\left\{\left(x_{2},-0.20\right),(0.5410,0.2828,0.1866)\right\}$ \\
\hline
\end{tabular}

Step 4. If the entire attribute weights are unreachable, therefore, according to step 2, the attributes' weighting vector is $\omega=(0.1651,0.1458,0.1706,0.1657,0.1782,0.1746)$. Then the weighted matrix $C=\left[c_{f z}\right]_{g \times s}$ can be obtained in Table 5 . 
Table 5. The weighted decision-making matrix $C$

\begin{tabular}{|c|c|c|c|}
\hline & $T_{1}$ & $T_{2}$ & $T_{3}$ \\
\hline$H_{1}$ & $\left\{\left(x_{0}, 0.45\right),(0.0896,0.8596,0.8127)\right\}$ & $\left\{\left(x_{0}, 0.47\right),(0.1150,0.9003,0.8218)\right\}$ & $\left\{\left(x_{0}, 0.26\right),(0.1485,0.8179,0.8877)\right\}$ \\
\hline$H_{2}$ & $\left\{\left(x_{0}, 0.28\right),(0.0560,0.8805,0.8447)\right\}$ & $\left\{\left(x_{0}, 0.26\right),(0.1004,0.8082,0.8407)\right\}$ & $\left\{\left(x_{1},-0.45\right),(0.0935,0.7629,0.7826)\right\}$ \\
\hline$H_{3}$ & $\left\{\left(x_{0}, 0.45\right),(0.0531,0.8435,0.8882)\right\}$ & $\left\{\left(x_{0}, 0.45\right),(0.1179,0.8403,0.7631)\right\}$ & $\left\{\left(x_{0}, 0.49\right),(0.1115,0.8800,0.8075)\right\}$ \\
\hline$H_{4}$ & $\left\{\left(x_{1},-0.34\right),(0.1757,0.7353,0.8026)\right\}$ & $\left\{\left(x_{1},-0.49\right),(0.1533,0.7519,0.7989)\right\}$ & $\left\{\left(x_{1},-0.32\right),(0.1593,0.6953,0.8304)\right\}$ \\
\hline$H_{5}$ & $\left\{\left(x_{1},-0.42\right),(0.0716,0.8837,0.8489)\right\}$ & $\left\{\left(x_{0}, 0.42\right),(0.0622,0.7852,0.8218)\right\}$ & $\left\{\left(x_{0}, 0.42\right),(0.0653,0.8877,0.8637)\right\}$ \\
\hline & $T_{4}$ & $T_{5}$ & $T_{6}$ \\
\hline$H_{1}$ & $\left\{\left(x_{0}, 0.47\right),(0.1527,0.9092,0.9665)\right\}$ & $\left\{\left(x_{1},-0.46\right),(0.0948,0.7323,0.9153)\right\}$ & $\left\{\left(x_{1},-0.37\right),(0.1017,0.7766,0.8373)\right\}$ \\
\hline$H_{2}$ & $\left\{\left(x_{0}, 0.41\right),(0.0787,0.7620,0.8253)\right\}$ & $\left\{\left(x_{0}, 0.45\right),(0.0468,0.8113,0.7819)\right\}$ & $\left\{\left(x_{0}, 0.22\right),(0.1395,0.8248,0.8287)\right\}$ \\
\hline$H_{3}$ & $\left\{\left(x_{1},-0.47\right),(0.1111,0.7951,0.7490)\right\}$ & $\left\{\left(x_{0}, 0.31\right),(0.0771,0.8096,0.7940)\right\}$ & $\left\{\left(x_{1},-0.41\right),(0.0945,0.7325,0.8378)\right\}$ \\
\hline$H_{4}$ & $\left\{\left(x_{1},-0.31\right),(0.0884,0.7834,0.7443)\right\}$ & $\left\{\left(x_{1},-0.24\right),(0.2019,0.7127,0.8173)\right\}$ & $\left\{\left(x_{1},-0.17\right),(0.1326,0.7536,0.8251)\right\}$ \\
\hline$H_{5}$ & $\left\{\left(x_{1},-0.35\right),(0.1624,0.8286,0.9036)\right\}$ & $\left\{\left(x_{1},-0.36\right),(0.0512,0.8630,0.8927)\right\}$ & $\left\{\left(x_{0}, 0.31\right),(0.1271,0.8021,0.7460)\right\}$ \\
\hline
\end{tabular}

Step 5. According to the weighted matrix $C=\left[c_{f z}\right]_{5 \times 6}$ and the types of attributes, the sum of benefit attributes $\Phi_{f(b)}$ and the sum of cost attributes $\Phi_{f(c)}$ are computed by Eq. (15) and (16) which listed in Table 6. (Suppose $T_{1}, T_{3}, T_{4}$ and $T_{6}$ are benefit attributes and $T_{2}$ as well as $T_{5}$ are cost attributes.)

Table 6. The calculated results of $\Phi_{f(b)}$ and $\Phi_{f(c)}$

\begin{tabular}{|c|c|c|}
\hline & $\Phi_{f(b)}$ & $\Phi_{f(c)}$ \\
\hline$H_{1}$ & $\left\{\left(x_{2},-0.19\right),(0.4100,0.4965,0.5838)\right\}$ & $\left\{\left(x_{1}, 0.02\right),(0.1989,0.6593,0.7522)\right\}$ \\
\hline$H_{2}$ & $\left\{\left(x_{1}, 0.46\right),(0.3216,0.4222,0.4521)\right\}$ & $\left\{\left(x_{1},-0.30\right),(0.1425,0.6556,0.6573)\right\}$ \\
\hline$H_{3}$ & $\left\{\left(x_{2}, 0.06\right),(0.3228,0.4323,0.4500)\right\}$ & $\left\{\left(x_{1},-0.24\right),(0.1859,0.6803,0.6059)\right\}$ \\
\hline$H_{4}$ & $\left\{\left(x_{2},-0.14\right),(0.4521,0.3018,0.4093)\right\}$ & $\left\{\left(x_{1}, 0.27\right),(0.3243,0.5359,0.6529)\right\}$ \\
\hline$H_{5}$ & $\left\{\left(x_{2},-0.04\right),(0.3655,0.5214,0.4942)\right\}$ & $\left\{\left(x_{1}, 0.06\right),(0.1103,0.6776,0.7337)\right\}$ \\
\hline
\end{tabular}

The score results of $\Phi_{f(b)}$ and $\Phi_{f(c)}$ are listed as:

$$
\begin{gathered}
\Phi_{f(b)}=\left\{\begin{array}{l}
e\left(\Phi_{1(b)}\right)=0.1337, e\left(\Phi_{2(b)}\right)=0.1173, e\left(\Phi_{3(b)}\right)=0.1645, \\
e\left(\Phi_{4(b)}\right)=0.2766, e\left(\Phi_{5(b)}\right)=0.1473 .
\end{array}\right\} ; \\
\Phi_{f(c)}=\left\{\begin{array}{l}
e\left(\Phi_{1(b)}\right)=0.0445, e\left(\Phi_{2(b)}\right)=0.0323, e\left(\Phi_{3(b)}\right)=0.0382, \\
e\left(\Phi_{4(b)}\right)=0.0800, e\left(\Phi_{5(b)}\right)=0.0413 .
\end{array}\right\} .
\end{gathered}
$$

Step 6. Figure up the $Q_{f}(f=1,2,3,4,5)$ of each alternative with Eq. (17);

$$
Q_{1}=0.1794, Q_{2}=0.1802, Q_{3}=0.2177, Q_{4}=0.3020, Q_{5}=0.1965 \text {. }
$$

Step 7. Compute the values of utility degree $U_{f}(f=1,2,3,4,5)$ based on each alternative's $Q_{f}$ $(f=1,2,3,4,5)$ by Eq. (18);

$$
U_{1}=59.41 \%, U_{2}=59.69 \%, U_{3}=72.09 \%, U_{4}=100.00 \%, U_{5}=65.09 \% \text {. }
$$


Step 8. In terms of the magnitude of $U_{f}$, the ordering of alternatives is: $H_{4}>H_{3}>H_{5}>$ $H_{2}>H_{1}$ and $H_{4}$ is second to none.

\subsection{Compare SVN2TLN-COPRAS method with aggregation operators}

In this section, we compare our proposed SVN2TLN-COPRAS method with SVN2TLWA operator, SVN2TLWG operator, SVN2TLHWA operator and SVN2TLHWG operator (Wu et al., 2018). Based on the attribute's weight and Table 4, the fused values by SVN2TLWA, SVN2TLWG, SVN2TLHWA and SVN2TLHWG operators are shown in Table 7.

Table 7. The fused values by using some existing operator

\begin{tabular}{|c|c|c|}
\hline & SVN2TLWA & SVN2TLWG \\
\hline$H_{1}$ & $\left\{\left(x_{3},-0.26\right),(0.5330,0.4354,0.4727)\right\}$ & $\left\{\left(x_{3},-0.35\right),(0.5190,0.5265,0.5571)\right\}$ \\
\hline$H_{2}$ & $\left\{\left(x_{3},-0.30\right),(0.5239,0.3803,0.4073)\right\}$ & $\left\{\left(x_{2}, 0.49\right),(0.4732,0.4882,0.4907)\right\}$ \\
\hline$H_{3}$ & $\left\{\left(x_{3}, 0.24\right),(0.4784,0.3843,0.4146)\right\}$ & $\left\{\left(x_{3}, 0.20\right),(0.4533,0.4765,0.5413)\right\}$ \\
\hline$H_{4}$ & $\left\{\left(x_{4},-0.46\right),(0.5117,0.2868,0.3687)\right\}$ & $\left\{\left(x_{3}, 0.34\right),(0.4618,0.5300,0.4769)\right\}$ \\
\hline$H_{5}$ & $\left\{\left(x_{3},-0.16\right),(0.5720,0.4563,0.4136)\right\}$ & $\left\{\left(x_{3},-0.25\right),(0.5209,0.5203,0.4757)\right\}$ \\
\hline & SVN2TLHWA & SVN2TLHWG \\
\hline$H_{1}$ & $\left\{\left(x_{3},-0.24\right),(0.5296,0.4538,0.4909)\right\}$ & $\left\{\left(x_{3},-0.32\right),(0.5225,0.5031,0.5363)\right\}$ \\
\hline$H_{2}$ & $\left\{\left(x_{3},-0.22\right),(0.5110,0.4019,0.4241)\right\}$ & $\left\{\left(x_{3},-0.43\right),(0.4844,0.4605,0.4682)\right\}$ \\
\hline$H_{3}$ & $\left\{\left(x_{3}, 0.27\right),(0.4722,0.4036,0.4405)\right\}$ & $\left\{\left(x_{3}, 0.22\right),(0.4593,0.4536,0.5104)\right\}$ \\
\hline$H_{4}$ & $\left\{\left(x_{4},-0.34\right),(0.4996,0.3220,0.3891)\right\}$ & $\left\{\left(x_{3}, 0.45\right),(0.4738,0.4638,0.4478)\right\}$ \\
\hline$H_{5}$ & $\left\{\left(x_{3},-0.11\right),(0.5607,0.4697,0.4271)\right\}$ & $\left\{\left(x_{3},-0.21\right),(0.5341,0.5037,0.4605)\right\}$ \\
\hline
\end{tabular}

According to the score of SVN2TLNs, we could derive the score results (Table 8).

Table 8. Score results of alternatives $H_{f}$

\begin{tabular}{|c|c|c|c|c|}
\hline & SVN2TLWA & SVN2TLWG & SVN2TLHWA & SVN2TLHWG \\
\hline$e\left(H_{1}\right)$ & 0.2471 & 0.2111 & 0.2431 & 0.2211 \\
\hline$e\left(H_{2}\right)$ & 0.2607 & 0.2063 & 0.2600 & 0.2223 \\
\hline$e\left(H_{3}\right)$ & 0.3019 & 0.2550 & 0.2959 & 0.2675 \\
\hline$e\left(H_{4}\right)$ & 0.3646 & 0.2701 & 0.3642 & 0.2997 \\
\hline$e\left(H_{5}\right)$ & 0.2690 & 0.2328 & 0.2669 & 0.2435 \\
\hline
\end{tabular}

The rankings of alternatives are collected in Table 9.

Table 9. The rankings of alternatives deriving from different methods

\begin{tabular}{|l|c|}
\hline & Order \\
\hline SVN2TLWA & $H_{4}>H_{3}>H_{5}>H_{2}>H_{1}$ \\
\hline SVN2TLWG & $H_{4}>H_{3}>H_{5}>H_{1}>H_{2}$ \\
\hline SVN2TLHWA & $H_{4}>H_{3}>H_{5}>H_{2}>H_{1}$ \\
\hline SVN2TLHWG & $H_{4}>H_{3}>H_{5}>H_{2}>H_{1}$ \\
\hline SVN2TLN-COPRAS model & $H_{4}>H_{3}>H_{5}>H_{2}>H_{1}$ \\
\hline
\end{tabular}


From the above calculation process and the final result summary Table 9, it's easy for us to conclude that the optimal scheme is consistent, but in a slightly different order.

According to the operational formulas of these operators, we can find that the SVN2TLWA operator and SVN2TLWG operator are incapable of taking the relationship between being fused variables into account so that the integration results in practical decision problems are too rough and imprecise. Moreover, the SVN2TLHWA operator and SVN2TLHWG operator adopt parameter control to consider the relationship between integrated variables, but their operation formula is extremely complex, which will lead to excessive workload in the actual decision making, so it is difficult to be applied.

Compared our proposed model with the above mentioned operators, although the decision results are slightly different, the SVN2TLN-COPRAS model has the precious merits in settling the inconsistent attributes by computing the utility degree shown as the percentage which depicts the alternative is better or worse than others, and the decision steps of this new model are very simple and more in line with the actual decision situation for consider the conflicting attributes.

\section{Conclusions}

In this paper, we develop the SVN2TLN-COPRAS model to solve the MAGDM and some basically concepts of SVN2TLSs. Firstly, we introduce the relevant knowledge about SVN2TLSs in a nutshell, such as the definition, the operation laws, a few of fused operators and so on. Then, combine the traditional COPRAS model with SVN2TLNs, and structure as well as elucidate the computing steps of the SVN2TLN-COPRAS pattern. Furthermore, in this article, we propose a method for determining attribute weights in different situations relying on the maximizing deviation method with SVN2TLNs. Last but not least, a numerical example about assessing the safety of construction project has been designed. And for further demonstrating the advantage of the new designed method, we also select a number of existed methods to have comparisons. In future research, we are committed to apply the SVN2TLNCOPRAS model to other fields so as to resolve more MAGDM problems.

\section{Acknowledgments}

The work was supported by the National Natural Science Foundation of China under Grant No. 71571128.

\section{References}

Atanassov, K. (1986). Intuitionistic fuzzy sets. Fuzzy Sets and Systems, 20(1), 87-96. https://doi.org/10.1016/S0165-0114(86)80034-3

Bekar, E. T., Cakmakci, M., \& Kahraman, C. (2016). Fuzzy COPRAS method for performance measurement in total productive maintenance: A comparative analysis. Journal of Business Economics and Management, 17(5), 663-684. https://doi.org/10.3846/16111699.2016.1202314

Chen, J. Q., \& Ye, J. (2017). Some Single-valued neutrosophic Dombi weighted aggregation operators for multiple attribute decision-making. Symmetry, 9(6), 82. https://doi.org/10.3390/sym9060082 
Fan, C. X., Fan, E., \& Ye, J. (2018). The cosine measure of single-valued neutrosophic multisets for multiple attribute decision-making. Symmetry, 10(5), 154. https://doi.org/10.3390/sym10050154

Garg, H. (2016). A new generalized Pythagorean fuzzy information aggregation using Einstein operations and its application to decision making. International Journal of Intelligent Systems, 31(9), 886-920. https://doi.org/10.1002/int.21809

Garg, H., \& Nancy. (2018). Linguistic single-valued neutrosophic prioritized aggregation operators and their applications to multiple-attribute group decision-making. Journal of Ambient Intelligence and Humanized Computing, 9, 1975-1997. https://doi.org/10.1007/s12652-018-0723-5

Gundogdu, F. K., \& Kahraman, C. (2019). Spherical fuzzy sets and spherical fuzzy TOPSIS method. Journal of Intelligent \& Fuzzy Systems, 36(1), 337-352. https://doi.org/10.3233/JIFS-181401

He, T. T., Wei, G. W., Lu, J. P., Wei, C., \& Lin, R. (2019). Pythagorean 2-tuple linguistic Taxonomy method for supplier selection in medical instrument industries. International Journal of Environmental Research and Public Health, 16(23), 4875. https://doi.org/10.3390/ijerph16234875

Herrera, F., \& Martinez, L. (2000). A 2-tuple fuzzy linguistic representation model for computing with words. IEEE Transactions on Fuzzy Systems, 8(6), 746-752. https://doi.org/10.1109/91.890332

Ju, D. W., Ju, Y. B., \& Wang, A. H. (2018). Multiple attribute group decision making based on Maclaurin symmetric mean operator under single-valued neutrosophic interval 2-tuple linguistic environment. Journal of Intelligent \& Fuzzy Systems, 34(4), 2579-2595. https://doi.org/10.3233/JIFS-17496

Liou, J. J. H., Tamosaitiene, J., Zavadskas, E. K., \& Tzeng, G. H. (2016). New hybrid COPRAS-G MADM Model for improving and selecting suppliers in green supply chain management. International Journal of Production Research, 54(1), 114-134. https://doi.org/10.1080/00207543.2015.1010747

Mahdiraji, H. A., Arzaghi, S., Stauskis, G., \& Zavadskas, E. K. (2018). A hybrid fuzzy BWM-COPRAS method for analyzing key factors of sustainable architecture. Sustainability, 10(5), 1626. https://doi.org/10.3390/su10051626

Matic, B., Jovanovic, S., Das, D. K., Zavadskas, E. K., Stevic, Z., Sremac, S., \& Marinkovic, M. (2019). A new hybrid MCDM model: Sustainable supplier selection in a construction company. Symmetry, 11(3), 353. https://doi.org/10.3390/sym11030353

Podvezko, V. (2011). The comparative analysis of MCDA methods SAW and COPRAS. Inzinerine Ekonomika-Engineering Economics, 22(2), 134-146. https://doi.org/10.5755/j01.ee.22.2.310

Roy, J., Sharma, H. K., Kar, S., Zavadskas, E. K., \& Saparauskas, J. (2019). An extended COPRAS model for multi-criteria decision-making problems and its application in web-based hotel evaluation and selection. Economic Research-Ekonomska Istrazivanja, 32(1), 219-253.

https://doi.org/10.1080/1331677X.2018.1543054

Sahin, R., \& Liu, P. D. (2016). Maximizing deviation method for neutrosophic multiple attribute decision making with incomplete weight information. Neural Computing \& Applications, 27, 2017-2029. https://doi.org/10.1007/s00521-015-1995-8

Smarandache, F. (1999). A unifying field in logics. Neutrosophy: Neutrosophic probability, set and logic. American Research Press.

Wang, H., Smarandache, F., Zhang, Y. Q., \& Sunderraman, R. (2010). Single valued neutrosophic sets. Multispace Multistruct, 4, 410-413.

Wang, J., Gao, H., \& Lu, M. (2019a). Approaches to strategic supplier selection under interval neutrosophic environment. Journal of Intelligent \& Fuzzy Systems, 37(2), 1707-1730. https://doi.org/10.3233/JIFS-179235

Wang, J., Wei, G. W., \& Lu, M. (2018a). TODIM method for multiple attribute group decision making under 2-tuple linguistic neutrosophic environment. Symmetry, 10(10), 486.

https://doi.org/10.3390/sym10100486 
Wang, J., Wei, G. W., Wei, C., \& Wu, J. (2020). Maximizing deviation method for multiple attribute decision making under q-rung orthopair fuzzy environment. Defence Technology, 16(5), 1073-1087. https://doi.org/10.1016/j.dt.2019.11.007

Wang, J., Wei, G. W., \& Wei, Y. (2018b). Models for green supplier selection with some 2-tuple linguistic neutrosophic number Bonferroni mean operators. Symmetry, 10(5), 131.

https://doi.org/10.3390/sym10050131

Wang, L., Zhang, H. Y., Wang, J. Q., \& Li, L. (2018c). Picture fuzzy normalized projection-based VIKOR method for the risk evaluation of construction project. Applied Soft Computing, 64, 216-226. https://doi.org/10.1016/j.asoc.2017.12.014

Wang, P., Wang, J., \& Wei, G. W. (2019b). EDAS method for multiple criteria group decision making under 2-tuple linguistic neutrosophic environment. Journal of Intelligent \& Fuzzy Systems, 37(2), 1597-1608. https://doi.org/10.3233/JIFS-179223

Wang, Y. M. (1998). Using the method of maximizing deviations to make decision for multi-indices. System Engineering and Electronics, 7, 24-26.

Wei, G. W., Wang, J., Lu, M., Wu, J., \& Wei, C. (2019a). Similarity measures of spherical fuzzy sets based on cosine function and their applications. IEEE Access, 7, 159069-159080. https://doi.org/10.1109/ACCESS.2019.2949296

Wei, G. W., Wu, J., Wei, C., Wang, J., \& Lu, J. P. (2019b). Models for MADM with 2-tuple linguistic neutrosophic Dombi Bonferroni mean operators. IEEE Access, 7, 108878-108905. https://doi.org/10.1109/ACCESS.2019.2930324

Wu, L. P., Gao, H., \& Wei, C. (2019). VIKOR method for financing risk assessment of rural tourism projects under interval-valued intuitionistic fuzzy environment. Journal of Intelligent \& Fuzzy Systems, 37(2), 2001-2008. https://doi.org/10.3233/JIFS-179262

Wu, L. P., Wei, G. W., Wu, J., \& Wei, C. (2020). Some interval-valued intuitionistic fuzzy Dombi Heronian mean operators and their application for evaluating the ecological value of forest ecological tourism demonstration areas. International Journal of Environmental Research and Public Health, 17(3), 829. https://doi.org/10.3390/ijerph17030829

Wu, Q., Wu, P., Zhou, L. G., Chen, H. Y., \& Guan, X. J. (2018). Some new Hamacher aggregation operators under single-valued neutrosophic 2-tuple linguistic environment and their applications to multi-attribute group decision making. Computers \& Industrial Engineering, 116, 144-162. https://doi.org/10.1016/j.cie.2017.12.024

Ye, J. (2014). Single valued neutrosophic cross-entropy for multicriteria decision making problems. Applied Mathematical Modelling, 38(3), 1170-1175. https://doi.org/10.1016/j.apm.2013.07.020

Yin, F. L., Lu, L., Chai, J. P., \& Yang, Y. B. (2016). Combination weighting method based on maximizing deviations and normalized constraint condition. International Journal of Security and Its Applications, 10(2), 39-50. https://doi.org/10.14257/ijsia.2016.10.2.04

Zadeh, L. A. (1965). Fuzzy sets. Information and Control, 8(3), 338-353. https://doi.org/10.1016/S0019-9958(65)90241-X

Zavadskas, E. K., Kaklauskas, A., \& Kvederyte, N. (2001). Multivariant design and multiple criteria analysis of a building life cycle. Informatica, 12(1), 169-188.

Zavadskas, E. K., Kaklauskas, A., \& Sarka, V. (1994). The new method of multi-criteria complex proportional assessment of projects. Technological and Economic Development of Economy, 3, 131-139.

Zhang, X. L., \& Xu, Z. S. (2014). Extension of TOPSIS to multiple criteria decision making with Pythagorean fuzzy sets. International Journal of Intelligent Systems, 29, 1061-1078.

https://doi.org/10.1002/int.21676 